\title{
On solving some functional equations
}

\section{Dmitry V Kruchinin*}

${ }^{*}$ Correspondence:
kruchinindm@gmail.com
Tomsk State University of Control
Systems and Radioelectronics
(TUSUR), Tomsk, Russia
National Research Tomsk
Polytechnic University (TPU), Tomsk,
Russia

${ }^{*}$ Correspondence: kruchinindm@gmail.com Systems and Radioelectronics (TUSUR), Tomsk, Russia Polytechnic University (TPU), Tomsk,

\begin{abstract}
Using the notion of composita and the Lagrange inversion theorem, we present techniques for solving the following functional equation $B(x)=H\left(x B(x)^{m}\right)$, where $H(x)$, $B(x)$ are generating functions and $m \in \mathbb{N}$. Also we give some examples.
\end{abstract}

MSC: Primary 05A15; secondary 65Q20; 39B12

Keywords: composita; generating function; Lagrange inversion theorem; functional equation

\section{Introduction}

In this paper we study the coefficients of the powers of an ordinary generating function and their properties. Using the notion of composita, we get the solution of the functional equation $B(x)=H\left(x B(x)^{m}\right)$, which is based on the Lagrange inversion equation, where $H(x), B(x)$ are generating functions and $m \in \mathbb{N}$.

In the papers [1-3], the author introduced the notion of composita of a given ordinary generating function $F(x)=\sum_{n>0} f(n) x^{n}$.

Suppose $F(x)=\sum_{n>0} f(n) x^{n}$ is the generating function, in which there is no free term $f(0)=0$. From this generating function we can write the following equation:

$$
[F(x)]^{k}=\sum_{n>0} F(n, k) x^{n}
$$

The expression $F(n, k)$ is the composita, and it is denoted by $F^{\Delta}(n, k)$.

Definition 1 The composita is the function of two variables defined by

$$
F^{\Delta}(n, k)=\sum_{\pi_{k} \in C_{n}} f\left(\lambda_{1}\right) f\left(\lambda_{2}\right) \cdots f\left(\lambda_{k}\right)
$$

where $C_{n}$ is a set of all compositions of an integer $n, \pi_{k}$ is the composition $\sum_{i=1}^{k} \lambda_{i}=n$ into $k$ parts exactly.

The expression $F^{\Delta}(n, k)$ takes a triangular form

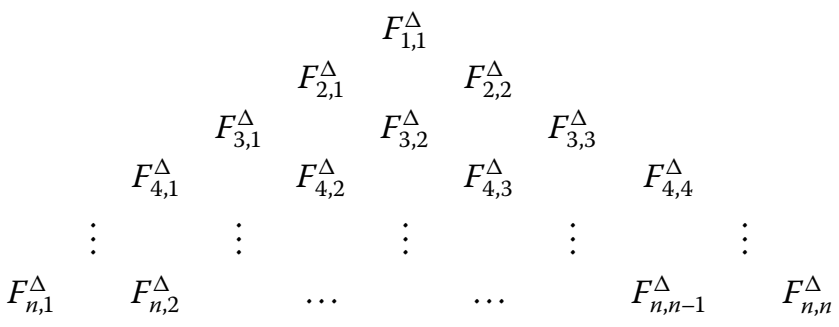

(0) 2015 Kruchinin; licensee Springer. This is an Open Access article distributed under the terms of the Creative Commons Attribution License (http://creativecommons.org/licenses/by/4.0), which permits unrestricted use, distribution, and reproduction in any medium, provided the original work is properly credited. 


\section{Lagrange inversion equation}

First we consider a solution of the functional equation

$$
A(x)=x H(A(x))
$$

where $A(x)$ and $H(x)$ are generating functions such that $H(x)=\sum_{n \geq 0} h(n) x^{n}$ and $A(x)=$ $\sum_{n>0} a(n) x^{n}$.

In the following lemma, we give the Lagrange inversion formula, which was proved by Stanley [4].

Lemma 2 (The Lagrange inversion formula) Suppose $H(x)=\sum_{n \geq 0} h(n) x^{n}$ with $h(0) \neq 0$, and let $A(x)$ be defined by

$$
A(x)=x H(A(x))
$$

Then

$$
n\left[x^{n}\right] A(x)^{k}=k\left[x^{n-k}\right] H(x)^{n}
$$

where $\left[x^{n}\right] A(x)^{k}$ is the coefficient of $x^{n}$ in $A(x)^{k}$ and $\left[x^{n-k}\right] H(x)^{n}$ is the coefficient of $x^{n-k}$ in $H(x)^{n}$.

By using the above Lemma 2, we now give the following theorem.

Theorem 3 Suppose $H(x)=\sum_{n \geq 0} h(n) x^{n}$ is a generating function, where $h(0) \neq 0, H_{x}^{\Delta}(n, k)$ is the composita of the generating function $x H(x)$, and $A(x)=\sum_{n>0} a(n) x^{n}$ is the generating function, which is obtained from the functional equation $A(x)=x H(A(x))$. Then the following condition holds true:

$$
A^{\Delta}(n, k)=\frac{k}{n} H_{x}^{\Delta}(2 n-k, n)
$$

Proof According to Lemma 2, for the solution of the functional equation $A(x)=x H(A(x))$, we can write

$$
n\left[x^{n}\right] A(x)^{k}=k\left[x^{n-k}\right] H(x)^{n} .
$$

On the left-hand side, there is the composita of the generating function $A(x)$ multiplied by $n$ :

$$
n\left[x^{n}\right] A(x)^{k}=n A^{\Delta}(n, k) .
$$

We know that

$$
(x H(x))^{k}=\sum_{n \geq k} H_{x}^{\Delta}(n, k) x^{n} .
$$


Then

$$
(H(x))^{k}=\sum_{n \geq k} H_{x}^{\Delta}(n, k) x^{n-k}
$$

If we replace $n-k$ by $m$, we obtain the following expression:

$$
(H(x))^{k}=\sum_{m \geq 0} H_{x}^{\Delta}(m+k, k) x^{m}
$$

Substituting $n$ for $k$ and $n-k$ for $m$, we get

$$
\left[x^{n-k}\right] H(x)^{n}=H_{x}^{\Delta}(2 n-k, n) .
$$

Therefore, we get

$$
A^{\Delta}(n, k)=\frac{k}{n} H_{x}^{\Delta}(2 n-k, n) .
$$

According to the above theorem, for solutions of the functional equation $A(x)=$ $x H(A(x))$, we can use the following expression:

$$
[A(x)]^{k}=\sum_{n \geq k} A^{\Delta}(n, k) x^{n}=\sum_{n \geq k} \frac{k}{n} H_{x}^{\Delta}(2 n-k, n) x^{n}
$$

where $H_{x}^{\Delta}(n, k)$ is the composita of the generating function $x H(x)$. Therefore,

$$
A(x)=\sum_{n \geq 1} \frac{1}{n} H_{x}^{\Delta}(2 n-1, n) x^{n} .
$$

Since the composita is uniquely determined by the generating function, formula (6) provides a solution of the inverse equation $A(x)=x H(A(x))$, when $A(x)$ is known and $H(x)$ is unknown. Hence,

$$
H_{x}^{\Delta}(n, k)=\frac{k}{2 k-n} A^{\Delta}(k, 2 k-n) .
$$

It should be noted that for $n=k$,

$$
H_{x}^{\Delta}(n, n)=A^{\Delta}(n, n)
$$

Next we give some examples of functional equations.

Example 4 Let us find an expression for coefficients of the generating function $A(x)=$ $\sum_{n>0} a(n) x^{n}$, which is defined by the functional equation

$$
A(x)=x+x A(x)+x A(x)^{2}+2 x A(x)^{3} .
$$

The generating function $x H(x)$ has the form

$$
x H(x)=x+x^{2}+x^{3}+2 x^{4} .
$$


The composita of $x H(x)$ is

$$
H_{x}^{\Delta}(n, k)=\sum_{j=0}^{k}\left(\begin{array}{l}
k \\
j
\end{array}\right) \sum_{i=j}^{n-k+j} 2^{n-3(k-j)-i}\left(\begin{array}{c}
j \\
i-j
\end{array}\right)\left(\begin{array}{c}
k-j \\
n-3(k-j)-i
\end{array}\right)
$$

According to formula (7), the coefficients of $A(x)$ are

$$
a(n)=\frac{1}{n} H_{x}^{\Delta}(2 n-1, n)
$$

Therefore, we get

$$
a(n)=\frac{1}{n} \sum_{j=0}^{n}\left(\begin{array}{l}
n \\
j
\end{array}\right) \sum_{i=j}^{n+j-1}\left(\begin{array}{c}
j \\
i-j
\end{array}\right) 2^{-n+3 j-i-1}\left(\begin{array}{c}
n-j \\
-n+3 j-i-1
\end{array}\right) .
$$

Example 5 Let us find an expression for coefficients of the generating function $B(x)=$ $\sum_{n \geq 0} a(n) x^{n}$, which is defined by the functional equation (see A064641 [5])

$$
B(x)=\frac{1-x-\sqrt{1-6 x-3 x^{2}}}{2 x(1+x)}
$$

Next we introduce the following generating function $A(x)=x B(x)$. Considering the functional equation, we can notice that

$$
A(x)=x \frac{1+A(x)+A(x)^{2}}{1-A(x)}
$$

Then we get the following functional equation:

$$
A(x)=x H(A(x))
$$

where $H(x)=\frac{1+x+x^{2}}{1-x}$.

Now we obtain the composita of $x H(x)$. The composita of $F(x)=x+x^{2}+x^{3}$ is

$$
F^{\Delta}(n, k)=\sum_{j=0}^{k}\left(\begin{array}{c}
j \\
n-k-j
\end{array}\right)\left(\begin{array}{l}
k \\
j
\end{array}\right) \text {. }
$$

The expression of coefficients of the generating function $[R(x)]^{k}=\left(\frac{1}{1-x}\right)^{k}$ is equal to

$$
\left(\begin{array}{c}
n+k-1 \\
k-1
\end{array}\right)
$$

Then we obtain the composita of $x H(x)$,

$$
H_{x}^{\Delta}(n, k)=\sum_{i=0}^{n-k}\left(\begin{array}{c}
k+i-1 \\
k-1
\end{array}\right) \sum_{j=0}^{k}\left(\begin{array}{c}
j \\
n-k-j-i
\end{array}\right)\left(\begin{array}{l}
k \\
j
\end{array}\right) .
$$


Hence, using Theorem 3, we get the composita of $A(x)$,

$$
A^{\Delta}(n, k)=\frac{k}{n} H_{x}^{\Delta}(2 n-k, n)=\frac{k}{n} \sum_{i=0}^{n-k}\left(\begin{array}{c}
n+i-1 \\
n-1
\end{array}\right) \sum_{j=0}^{n}\left(\begin{array}{c}
j \\
n-k-j-i
\end{array}\right)\left(\begin{array}{l}
n \\
j
\end{array}\right) .
$$

The coefficients of $B(x)$ are

$$
b(n)=A^{\Delta}(n+1,1) .
$$

Therefore, we obtain

$$
b(n)=\frac{1}{n+1} \sum_{i=0}^{n}\left(\begin{array}{c}
n+i \\
n
\end{array}\right) \sum_{j=0}^{n+1}\left(\begin{array}{c}
j \\
n-j-i
\end{array}\right)\left(\begin{array}{c}
n+1 \\
j
\end{array}\right) .
$$

\section{The generalized Lagrange inversion equation}

Next we generalize the case $A(x)=x H(A(x))$.

Replacing $A(x)$ by $x B(x)$ in the functional equation (4), we get

$$
B(x)=H(x B(x)) .
$$

Let us introduce the following definitions.

Definition 6 The left composita of the generating function $B(x)$ in the functional equation (8) is the composita

$$
H_{x}^{\Delta}(n, k)=\frac{k}{2 k-n} B_{x}^{\Delta}(k, 2 k-n)
$$

where $H_{x}^{\Delta}(n, k)$ is the composita of the generating function $x B(x)$.

Definition 7 The right composita of the generating function $H(x)$ in the functional equation (8) is the composita

$$
B_{x}^{\Delta}(n, k)=\frac{k}{n} H_{x}^{\Delta}(2 n-k, n),
$$

where $H_{x}^{\Delta}(n, k)$ is the composita of the generating function $x H(x)$.

There exists the left composita for every left composita and there exists the right composita for every right composita.

Formula (6) can be generalized for the case when a generating function is the solution of a certain functional equation. Let us prove the following theorem.

Theorem 8 Suppose $H(x)=\sum_{n \geq 0} h(n) x^{n}$ and $B(x)=\sum_{n \geq 0} b(n) x^{n}$ are generating functions such that $B(x)=H\left(x B(x)^{m}\right)$, where $m \in \mathbb{N} ; H_{x}^{\Delta}(n, k)$ and $B_{x}^{\Delta}(n, k)$ are the compositae of the generating functions $x H(x)$ and $x B(x)$, respectively. Then

$$
B_{x}^{\Delta}(n, k)=\frac{k}{i_{m-1}} H_{x}^{\Delta}\left(i_{m}, i_{m-1}\right),
$$

where $i_{m}=(m+1) n-m k$. 
Proof For $m=0$, we have

$$
B(x)=H\left(x B(x)^{0}\right)=G(x), \quad i_{m-1}=k, i_{m}=n .
$$

Then we obtain the identity

$$
B_{x}^{\Delta}(n, k)=\frac{k}{k} H_{x}^{\Delta}(n, k) .
$$

For $m=1$, we have

$$
B_{1}(x)=G\left(x B_{1}(x)\right), \quad i_{m-1}=n, i_{m}=2 n-k .
$$

Then we obtain

$$
B_{x, 1}^{\Delta}(n, k)=\frac{k}{n} H_{x}^{\Delta}(2 n-k, n)
$$

that satisfy Theorem 6 .

By induction, we put that for $m$ the solution of the equation

$$
B_{m}(x)=H\left(x B_{m}(x)^{m}\right)
$$

is

$$
B_{x, m}^{\Delta}(n, k)=\frac{k}{i_{m-1}} H_{x}^{\Delta}\left(i_{m}, i_{m-1}\right) .
$$

Then we find the solution for $m+1$,

$$
B_{m+1}(x)=H\left(x B_{m+1}(x)^{m+1}\right) .
$$

For this purpose, we consider the following functional equation:

$$
B_{m+1}(x)=B_{m}\left(x B_{m+1}(x)\right) .
$$

Instead of $B_{m}(x)$, we substitute the right hand-side of (10)

$$
B_{m+1}(x)=H\left(x B_{m+1}(x)\left[B_{m}\left(x B_{m+1}(x)\right)\right]^{m}\right) ;
$$

from whence it follows that

$$
B_{m+1}(x)=H\left(x B_{m+1}(x)^{m+1}\right) .
$$

We note that $B_{x, m+1}^{\Delta}(n, k)$ is the right composita of $B_{m}(x)$,

$$
B_{x, m+1}^{\Delta}(n, k)=\frac{k}{n} B_{x, m}^{\Delta}(2 n-k, n)
$$

where $B_{x, m}^{\Delta}(n, k)$ is the composita of the generating function $x B_{m}(x)$. 
Table 1 Table of functional equations

\begin{tabular}{llll}
\hline Equation & Function $\boldsymbol{B}(\boldsymbol{x})$ & Composita $\boldsymbol{B}_{\boldsymbol{x}}^{\boldsymbol{\Delta}}(\boldsymbol{n}, \boldsymbol{k})$ & OEIS \\
\hline$B(x)=1+x B^{0}(x)$ & $1+x$ & $\left(\begin{array}{c}k \\
n-k\end{array}\right)$ & \\
$B(x)=1+x B^{1}(x)$ & $\frac{1}{1-x}$ & $\left(\begin{array}{c}n-1 \\
k-1\end{array}\right)$ & $\mathrm{A} 000012$ \\
$B(x)=1+x B^{2}(x)$ & $\frac{1-\sqrt{1-4 x}}{2 x}$ & $\frac{k}{n}\left(\begin{array}{c}2 n-k-1 \\
n-1\end{array}\right)$ & $\mathrm{A} 000108$ \\
$B(x)=1+x B^{3}(x)$ & & $\frac{k}{n}\left(\begin{array}{c}3 n-2 k \\
n-k\end{array}\right)$ & $\mathrm{A} 001764$ \\
\hline
\end{tabular}

Then

$$
B_{x, m+1}^{\Delta}(n, k)=\frac{k}{(m+1) n-m k} H_{x}^{\Delta}((m+2) n-(m+1) k,(m+1) n-m k) .
$$

Therefore, for the functional equation

$$
B_{m+1}(x)=B_{m}\left(x B_{m+1}(x)\right),
$$

we obtain the required condition

$$
B_{x, m+1}^{\Delta}(n, k)=\frac{k}{i_{m}} H_{x}^{\Delta}\left(i_{m+1}, i_{m}\right)
$$

where $i_{m}=(m+1) n-m k$.

In Table 1 we present a sequence of functional equations for the generating function $H(x)=1+x$, where OEIS means the On-Line Encyclopedia of Integer Sequences [5].

Competing interests

The author declares that they have no competing interests.

\section{Acknowledgements}

The author wishes to thank the referees for their useful comments. This work was partially supported by the Ministry of Education and Science of Russia, government order No. 1220 'Theoretical bases of designing informational-safe systems'.

Received: 30 October 2014 Accepted: 25 December 2014 Published online: 30 January 2015

\section{References}

1. Kruchinin, W, Kruchinin, DV: Composita and its properties. J. Anal. Number Theory 2(2), 1-8 (2014)

2. Kruchinin, DV, Kruchinin, W: A method for obtaining generating function for central coefficients of triangles. J. Integer Seq. 15, 1-10 (2012)

3. Kruchinin, DV, Kruchinin, W: Application of a composition of generating functions for obtaining explicit formulas of polynomials. J. Math. Anal. Appl. 404, 161-171 (2013)

4. Stanley, RP: Enumerative Combinatorics, vol. 2. Cambridge Studies in Advanced Mathematics. Cambridge University Press, Cambridge (1999)

5. Sloane, JA: The On-Line Encyclopedia of Integer Sequences. www.oeis.org 